\title{
Production technology and application of thin film materials in micro fabrication
}

\author{
Xiaoming $\mathrm{Hu}^{1}$ \\ ${ }^{1}$ Dept. electronic \& electrical engineering, Wuhan railway vocational college of technology, \\ Wuhan, Chain \\ Email: huxiaolili@tom.com, Wuhan jiangxia district hidden dragon road, no. 1,430205
}

Keywords: thin film, micro manufacturing, Physical vapor deposition, Chemical vapor deposition

\begin{abstract}
This paper introduces the film material in micro manufacturing in general, it is covered in a wafer. Then, it focuses on the production process of the thin film, mainly has the PVD method, which is formed by the physical method; the other is VCD, that is the chemical method to produce the film. Finally, the various uses of micro thin films are introduced.
\end{abstract}

\section{Brief introduction for thin film materials}

A thin film is a layer of material ranging from fractions of a nanometer (monolayer) to several micrometers in thickness. Electronic semiconductor devices and optical coatings are the main applications benefiting from thin-film construction. The thin-film is on a wafer.

A typical wafer is made out of extremely pure silicon that is grown into mono-crystalline cylindrical ingots (boules) up to $300 \mathrm{~mm}$ (slightly less than 12 inches) in diameter using the Czochralski process. These ingots are then sliced into wafers about $0.75 \mathrm{~mm}$ thick and polished to obtain a very regular and flat surface.

The act of applying a thin film to a surface is thin-film deposition - any technique for depositing a thin film of material onto a substrate or wafer. "Thin" is a relative term, but most deposition techniques control layer thickness within a few tens of nanometres. Molecular beam epitaxy allows a single layer of atoms to be deposited at a time.

The performance of optical coatings (e.g., antireflective, or AR, coatings) are typically enhanced when the thin-film coating consists of multiple layers having varying thicknesses and refractive indices. Alternatively, with the hybrid nanostructures of dielectric nanoparticles and dieletric coating, the optically incoupling can also be further enhanced. ${ }^{[1]}$ Similarly, a periodic structure of alternating thin films of different materials may collectively form a so-called superlattice which exploits the phenomenon of quantum confinement by restricting electronic phenomena to twodimensions.

It is useful in the manufacture of optics (for reflective, anti-reflective coatings or self-cleaning glass, for instance), electronics (layers of insulators, semiconductors, and conductors form integrated circuits), packaging (i.e., aluminium-coated PET film), and in contemporary art . Similar processes are sometimes used where thickness is not important: for instance, the purification of copper by electroplating, and the deposition of silicon and enriched uranium by a CVD-like process after gas-phase processing. Deposition techniques fall into two broad categories, depending on whether the process is primarily chemical or physical. ${ }^{[2]}$ Many toxic materials are used in the fabrication process. ${ }^{[3]}$ These include: poisonous elemental dopants, such as arsenic, antimony, and phosphorus.poisonous compounds, such as arsine, phosphine, and silane.highly reactive liquids, such as hydrogen peroxide, fuming nitric acid, sulfuric acid, and hydrofluoric acid.

\section{Thin film production process}

\subsection{Physical vapor deposition (PVD)}

Physical vapor deposition (PVD) describes a variety of vacuum deposition methods which can be used to produce thin films. PVD uses physical process (such as heating or sputtering) to produce 
a vapor of material, which is then deposited on the object which requires coating. PVD is used in the manufacture of items which require thin films for mechanical, optical, chemical or electronic functions. Examples include semiconductor devices such as thin film solar panels, ${ }^{[4]}$ aluminized PET film for food packaging and balloons, and coated cutting tools for metalworking. ${ }^{[6]}$ Besides PVD tools for fabrication, special smaller tools (mainly for scientific purposes) have been developed.Common industrial coatings applied by PVD are titanium nitride, zirconium nitride, chromium nitride, titanium aluminum nitride. ${ }^{[7]}$ The source material is unavoidably also deposited on most other surfaces interior to the vacuum chamber, including the fixturing used to hold the parts.The material to be deposited is placed in an energetic, entropic environment, so that particles of material escape its surface. Facing this source is a cooler surface which draws energy from these particles as they arrive, allowing them to form a solid layer. The whole system is kept in a vacuum deposition chamber, to allow the particles to travel as freely as possible. Since particles tend to follow a straight path, films deposited by physical means are commonly directional, rather than conformal.

Examples of physical deposition include: A thermal evaporator that uses an electric resistance heater to melt the material and raise its vapor pressure to a useful range. This is done in a high vacuum, both to allow the vapor to reach the substrate without reacting with or scattering against other gas-phase atoms in the chamber, and reduce the incorporation of impurities from the residual gas in the vacuum chamber. Obviously, only materials with a much higher vapor pressure than the heating element can be deposited without contamination of the film. Molecular beam epitaxy is a particularly sophisticated form of thermal evaporation.An electron beam evaporator fires a highenergy beam from an electron gun to boil a small spot of material; since the heating is not uniform, lower vapor pressure materials can be deposited. The beam is usually bent through an angle of $270^{\circ}$ in order to ensure that the gun filament is not directly exposed to the evaporant flux. Typical deposition rates for electron beam evaporation range from 1 to 10 nanometres per second. In molecular beam epitaxy (MBE), slow streams of an element can be directed at the substrate, so that material deposits one atomic layer at a time. Compounds such as gallium arsenide are usually deposited by repeatedly applying a layer of one element (i.e., gallium), then a layer of the other (i.e., arsenic), so that the process is chemical, as well as physical. The beam of material can be generated by either physical means (that is, by a furnace) or by a chemical reaction (chemical beam epitaxy).

Sputtering relies on a plasma (usually a noble gas, such as argon) to knock material from a "target" a few atoms at a time. The target can be kept at a relatively low temperature, since the process is not one of evaporation, making this one of the most flexible deposition techniques. It is especially useful for compounds or mixtures, where different components would otherwise tend to evaporate at different rates. Note, sputtering's step coverage is more or less conformal. It is also widely used in the optical media. The manufacturing of all formats of CD, DVD, and BD are done with the help of this technique. It is a fast technique and also it provides a good thickness control. Presently, nitrogen and oxygen gases are also being used in sputtering.Pulsed laser deposition systems work by an ablation process. Pulses of focused laser light vaporize the surface of the target material and convert it to plasma; this plasma usually reverts to a gas before it reaches the substrate. Cathodic arc deposition (arc-PVD) which is a kind of ion beam deposition where an electrical arc is created that literally blasts ions from the cathode. The arc has an extremely high power density resulting in a high level of ionization (30-100\%), multiply charged ions, neutral particles, clusters and macro-particles (droplets). If a reactive gas is introduced during the evaporation process, dissociation, ionization and excitation can occur during interaction with the ion flux and a compound film will be deposited. Electrohydrodynamic deposition (electrospray deposition) is a relatively new process of thin film deposition. The liquid to be deposited, either in the form of nano-particle solution or simply a solution, is fed to a small capillary nozzle (usually metallic) which is connected to a high voltage. The substrate on which the film has to be deposited is connected to ground. Through the influence of electric field, the liquid coming out of the nozzle takes a conical shape (Taylor cone) and at the apex of the cone a thin jet emanates which disintegrates into very fine and small positively charged droplets under the influence of Rayleigh 
charge limt. The droplets keep getting smaller and smaller and ultimately get deposited on the substrate as a uniform thin layer.

\subsection{Chemical vapor deposition (CVD)}

Chemical vapor deposition (CVD) is a chemical process used to produce high quality, highperformance, solid materials. The process is often used in the semiconductor industry to produce thin films. In typical CVD, the wafer (substrate) is exposed to one or more volatile precursors, which react and/or decompose on the substrate surface to produce the desired deposit. Frequently, volatile by-products are also produced, which are removed by gas flow through the reaction chamber.CVD is practiced in a variety of formats. These processes generally differ in the means by which chemical reactions are initiated.Classified by operating pressure:Atmospheric pressure CVD (APCVD) - CVD at atmospheric pressure.Low-pressure CVD (LPCVD) - CVD at sub-atmospheric pressures. ${ }^{[8]}$ Reduced pressures tend to reduce unwanted gas-phase reactions and improve film uniformity across the wafer.Ultrahigh vacuum CVD (UHVCVD) - CVD at very low pressure, typically below $10^{-6} \mathrm{~Pa}\left(\sim 10^{-8}\right.$ torr). Note that in other fields, a lower division between high and ultra-high vacuum is common, often $10^{-7}$ Pa.Most modern CVD is either LPCVD or UHVCVD.Classified by physical characteristics of vapor:Aerosol assisted CVD (AACVD) - CVD in which the precursors are transported to the substrate by means of a liquid/gas aerosol, which can be generated ultrasonically. This technique is suitable for use with non-volatile precursors.Direct liquid injection CVD (DLICVD) - CVD in which the precursors are in liquid form (liquid or solid dissolved in a convenient solvent). Liquid solutions are injected in a vaporization chamber towards injectors (typically car injectors). The precursor vapors are then transported to the substrate as in classical CVD. This technique is suitable for use on liquid or solid precursors. High growth rates can be reached using this technique.

Plasma methods :Plasma-Enhanced CVD (PECVD) - CVD that utilizes plasma to enhance chemical reaction rates of the precursors. ${ }^{[9]}$ PECVD processing allows deposition at lower temperatures, which is often critical in the manufacture of semiconductors. The lower temperatures also allow for the deposition of organic coatings, such as plasma polymers, that have been used for nanoparticle surface functionalization. ${ }^{[10]}$ Remote plasma-enhanced CVD (RPECVD) - Similar to PECVD except that the wafer substrate is not directly in the plasma discharge region. Removing the wafer from the plasma region allows processing temperatures down to room temperature.Atomiclayer CVD (ALCVD) - Deposits successive layers of different substances to produce layered, crystalline films. See Atomic layer epitaxy.Combustion Chemical Vapor Deposition (CCVD) Combustion Chemical Vapor Deposition or flame pyrolysis is an open-atmosphere, flame-based technique for depositing high-quality thin films and nanomaterials.

Hot filament CVD (HFCVD) - also known as catalytic CVD (Cat-CVD) or more commonly, initiated CVD (iCVD), this process uses a hot filament to chemically decompose the source gases. ${ }^{[11]}$ The filament temperature and substrate temperature thus are independently controlled, allowing colder temperatures for better adsorption rates at the substrate and higher temperatures necessary for decomposition of precursors to free radicals at the filament. ${ }^{[12]}$

Hybrid Physical-Chemical Vapor Deposition (HPCVD) - This process involves both chemical decomposition of precursor gas and vaporization of a solid source.Metalorganic chemical vapor deposition (MOCVD) - This CVD process is based on metalorganic precursors.Rapid thermal CVD (RTCVD) - This CVD process uses heating lamps or other methods to rapidly heat the wafer substrate. Heating only the substrate rather than the gas or chamber walls helps reduce unwanted gas-phase reactions that can lead to particle formation.Vapor-phase epitaxy (VPE)Photo-initiated CVD (PICVD) - This process uses UV light to stimulate chemical reactions. It is similar to plasma processing, given that plasmas are strong emitters of UV radiation. Under certain conditions, PICVD can be operated at or near atmospheric pressure. ${ }^{[13]}$

\subsection{Molecular beam epitaxy}

Molecular beam epitaxy (MBE) is one of several methods of depositing single crystals. It was invented in the late 1960s at Bell Telephone Laboratories by J. R. Arthur and Alfred Y. Cho. ${ }^{[14]}$ 
Method:Molecular beam epitaxy takes place in high vacuum or ultra-high vacuum $\left(10^{-8} \mathrm{~Pa}\right)$. The most important aspect of MBE is the deposition rate (typically less than $3000 \mathrm{~nm}$ per hour) that allows the films to grow epitaxially. These deposition rates require proportionally better vacuum to achieve the same impurity levels as other deposition techniques. The absence of carrier gases as well as the ultra high vacuum environment result in the highest achievable purity of the grown films.In solid-source MBE, elements such as gallium and arsenic, in ultra-pure form, are heated in separate quasi-Knudsen effusion cells until they begin to slowly sublime. The gaseous elements then condense on the wafer, where they may react with each other. In the example of gallium and arsenic, single-crystal gallium arsenide is formed. The term "beam" means that evaporated atoms do not interact with each other or vacuum chamber gases until they reach the wafer, due to the long mean free paths of the atoms.

During operation, reflection high energy electron diffraction (RHEED) is often used for monitoring the growth of the crystal layers. A computer controls shutters in front of each furnace, allowing precise control of the thickness of each layer, down to a single layer of atoms. Intricate structures of layers of different materials may be fabricated this way. Such control has allowed the development of structures where the electrons can be confined in space, giving quantum wells or even quantum dots. Such layers are now a critical part of many modern semiconductor devices, including semiconductor lasers and light-emitting diodes.

In systems where the substrate needs to be cooled, the ultra-high vacuum environment within the growth chamber is maintained by a system of cryopumps, and cryopanels, chilled using liquid nitrogen or cold nitrogen gas to a temperature close to 77 Kelvin ( -196 degrees Celsius). Cryogenic temperatures act as a sink for impurities in the vacuum, so vacuum levels need to be several orders of magnitude better to deposit films under these conditions. In other systems, the wafers on which the crystals are grown may be mounted on a rotating platter which can be heated to several hundred degrees Celsius during operation.Molecular beam epitaxy is also used for the deposition of some types of organic semiconductors. In this case, molecules, rather than atoms, are evaporated and deposited onto the wafer. Other variations include gas-source MBE, which resembles chemical vapor deposition.Lately molecular beam epitaxy has been used to deposit oxide materials for advanced electronic, magnetic and optical applications. For these purposes, MBE systems have to be modified to incorporate oxygen sources. ${ }^{[15]}$

Atomic layer deposition (ALD) is a thin film deposition technique that is based on the sequential use of a gas phase chemical process. The majority of ALD reactions use two chemicals, typically called precursors. These precursors react with the surface of a material one at a time in a sequential, self-limiting, manner. Through the repeated exposure to separate precursors, a thin film is slowly deposited.Atomic Layer Deposition (ALD) is a thin film deposition method in which a film is grown on a substrate by exposing its surface to alternate gaseous species (typically referred to as precursors). In contrast to chemical vapor deposition, the precursors are never present simultaneously in the reactor, but they are inserted as a series of sequential, non-overlapping pulses. In each of these pulses the precursor molecules react with the surface in a self-limiting way, so that the reaction terminates once all the reactive sites on the surface are consumed. Consequently, the maximum amount of material deposited on the surface after a single exposure to all of the precursors (a so-called ALD cycle) is determined by the nature of the precursor-surface interaction. By varying the number of cycles it is possible to grow materials uniformly and with high precision on arbitrarily complex and large substrates. 

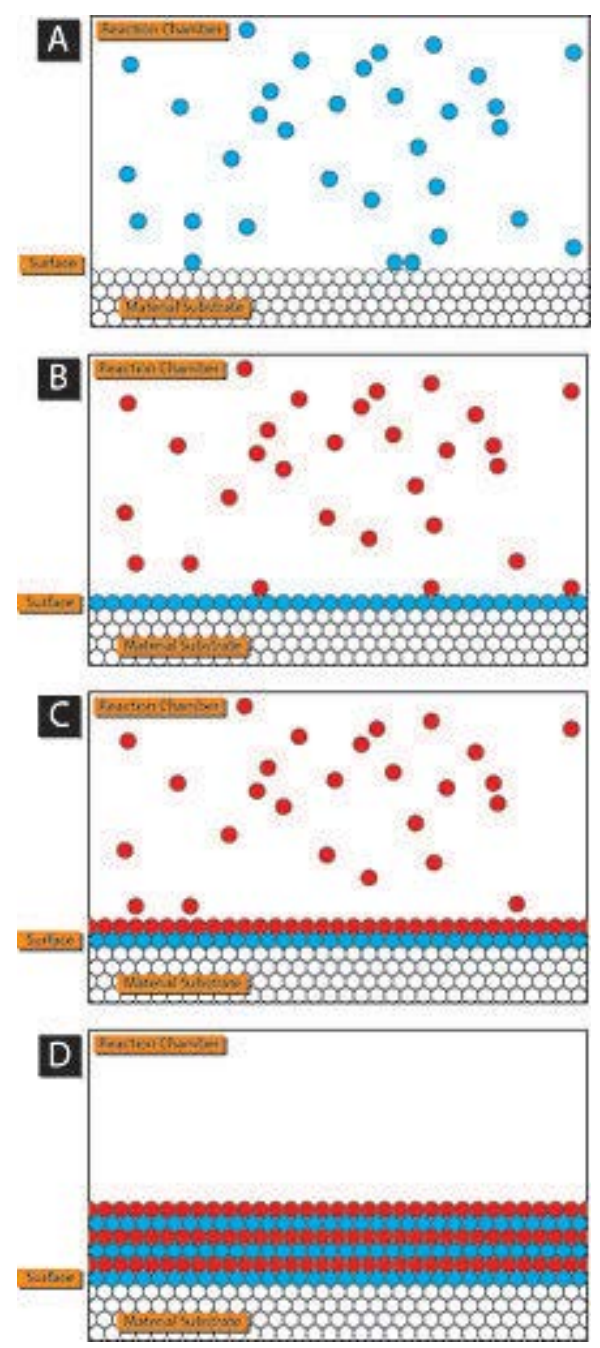

Fig.1. ALD coated process

A basic schematic of the Atomic Layer Deposition (ALD) process. See Fig.1. In Frame A, precursor 1 (in blue) is added to the reaction chamber containing the material surface to be coated by ALD. After precursor 1 has adsorbed on the surface, any excess is removed from the reaction chamber. Precursor 2 (red) is added (Frame B) and reacts with precursor 1 to create another layer on the surface (Frame C). Precursor 2 is then cleared from the reaction chamber and this process is repeated until a desired thickness is achieved and the resulting product resembles Frame D.ALD is an active field of research, with hundreds of different processes published in the scientific literature, though some of them exhibit behaviors that depart from that of an ideal ALD process. ${ }^{[16]}$

\section{Application}

\subsection{Thin-film photovoltaic cells}

Thin-film technologies are also being developed as a means of substantially reducing the cost of solar cells. The rationale for this is thin film solar cells are cheaper to manufacture owing to their reduced material costs, energy costs, handling costs and capital costs. This is especially represented in the use of printed electronics (roll-to-roll) processes. Other thin-film technologies, that are still in an early stage of ongoing research or with limited commercial availability, are often classified as emerging or third generation photovoltaic cells and include, organic, dye-sensitized, and polymer solar cells, as well as quantum dot, copper zinc tin sulfide, nanocrystal and perovskite solar cells.

\subsection{Thin film sensor}

Thin film sensor - A palladium thin film sensor is based on an opposing property that depends on the nanoscale structures within the thin film. In the thin film, nanosized palladium particles swell when the hydride is formed, and in the process of expanding, some of them form new electrical 
connections with their neighbors. The resistance decreases because of the increased number of conducting pathways.

\subsection{The others}

MBE is widely used in the manufacture of semiconductor devices, including transistors for cellular phones and WiFi. As mentioned previously, PVD coatings are generally used to improve hardness, wear resistance and oxidation resistance. Thus, such coatings use in a wide range of applications such as: Aerospace,Automotive,Surgical/Medical ,Dies and moulds for all manner of material processing,Cutting tools,Firearms, Optics, Watches,Thin films (window tint, food packaging, etc.),Darts barrels,Metals (Aluminum, Copper, Bronze, etc.)

\section{References}

[1].Optically enhanced semi-transparent organic solar cells through hybrid metal /nanoparticle/ http: //dx.doi.org/10.1016/j.nanoen.2015.08.014

[2].Functional Polymer Films Eds. R. Advincula and W. Knoll - Wiley, 2011, ISBN 9783527321902.

[3].CNET. “Why tech pollution's going global.” April 25, 2002. Retrieved November 9, 2015.

[4].Selvakumar, N.; Barshilia, Harish C. (2012-03-01). "Review of physical vapor deposited (PVD) spectrally selective coatings for mid- and high-temperature solar thermal applications". Solar Energy Materials and Solar Cells 98: 1-23. doi:10.1016/j.solmat.2011.10.028.

[5]."Product Development | Coating Services Group". coatingservicesgroup.com. Retrieved 201510-09.

[6].Fortunato,E.;Barquinha, P.; Martins, R. (2012-06-12). "Oxide Semiconductor Thin-Film Transistors: A Review of Recent Advances". Advanced Materials 24 (22): 2945-2986. doi:10.1002/adma.201103228. ISSN 1521-4095.

[7]. http://www.coatingservicesgroup.com

[8]."Low Pressure Chemical Vapor Deposition - Technology and Equipment". Crystec Technology Trading GmbH.

[9].Crystec Technology Trading GmbH, Plasma Enhanced Chemical Vapor Deposition Technology and Equipment

[10].Tavares, Jason; Swanson, E.J.; Coulombe, S. (2008). "Plasma Synthesis of Coated Metal Nanoparticles with Surface Properties Tailored for Dispersion". Plasma Processes and Polymers 5 (8): 759. doi:10.1002/ppap.200800074.

[11].Schropp, R.E.I.; B. Stannowski; A.M. Brockhoff; P.A.T.T. van Veenendaal; J.K. Rath. "Hot wire CVD of heterogeneous and polycrystalline silicon semiconducting thin films for application in thin film transistors and solar cells" (PDF). Materials Physics and Mechanics. pp. 73-82.

[12].Gleason, Karen K.; Kenneth K.S. Lau; Jeffrey A. Caulfield (2000). "Structure and Morphology of Fluorocarbon Films Grown by Hot Filament Chemical Vapor Deposition". Chemistry of Materials 12 (10): 3032. doi:10.1021/cm000499w.

[13].Dorval Dion, C.A.; Tavares, J.R. (2013). "Photo-Initiated Chemical Vapour Deposition as a Scalable Particle Functionalization Technology (A Practical Review)". Powder Technology. doi:10.1016/j.powtec.2013.02.024.

[14].Cho, A. Y.; Arthur, J. R.; Jr (1975). "Molecular beam epitaxy". Prog. Solid State Chem. 10: 157-192. doi:10.1016/0079-6786(75)90005-9.

[15].J. Cheng, V.K. Lazarov, et al, J. of Vacuum Science \& Technology B, 27, 148(2009). 
[16].Puurunen, Riikka. Surface chemistry of atomic layer deposition: A case study for the trimethylaluminum/water process, Journal of Applied Physics 97 , 121301 (2005).and http://wikipedia./file:ALD. 